\title{
THE MANAGEMENT OF EPILEPSY
}

D Smith, D Chadwick

$\mathrm{E}$ pilepsy is a common, sometimes chronic, condition with physical risks and psychological and socioeconomic consequences which impair quality of life. The management of patients with epilepsy demands long term commitment from both the general practitioner (GP) and the specialist.

The prime requirements are a complete diagnosis, selection of optimal treatment, and counselling appropriate to individual needs. The majority of patients will enter remission and may be discharged to the care of their GP, while the remainder need continued care in the specialist clinic.

In the course of the condition the patient (and carer/family) should be sufficiently well informed to make decisions about choices of treatment, the need for long term treatment, and options for dealing with the drug resistant condition and its consequences.

Ideally this process will involve cooperation between the consultant and nurse specialist and the primary care physician. While, at present, these facilities are not widely available, this article focuses on this model of care.

\section{STARTING TREATMENT}

The decision to start treatment should not be taken lightly. ${ }^{1}$ It represents a balance between the likelihood of further seizures with their attendant risks, including the small but real risk of sudden unexpected death (SUDEP), ${ }^{2}$ and the consequences, inconvenience, and risks of taking regular medication for each individual.

\section{Prophylactic treatment}

Prophylactic treatment has sometimes been advocated, notably in patients with severe head injury. While immediate treatment may reduce the risk of early post-traumatic seizures (within one week of injury) it does not influence the risk of late post-traumatic epilepsy. ${ }^{3}$ Studies addressing this issue in other neurological conditions with a high prospective risk of epilepsy (febrile seizures, craniotomy, cerebral tumours) have failed to show any evidence of benefit.

\section{Single seizures}

Patients presenting with a first seizure, where avoidable provocative factors have been excluded, represent a common clinical dilemma. Methodological differences explain the widely varying estimates of risk of recurrence. Meta-analysis of prospective studies indicate an overall two year risk of $30-40 \%$. The lowest risk (24\%) is in patients with no identified cause who have a normal electroencephalogram (EEG), and the highest risk (65\%) is in those with a remote neurological insult and an epileptiform EEG. ${ }^{4}$

Treatment after a first tonic-clonic seizure halves the two year risk of seizures from approximately $40 \%$ to $20 \% .^{5}$ However, this is not associated with any improvement in longer term outcomes such as proportions of patients achieving a one year remission.

While most neurologists do not advocate treatment routinely, patients who have a high risk of recurrence which would have significant social implications should be given the option and may elect to start treatment.

\section{Recurrent seizures}

The decision to start treatment is much more straightforward in a patient with recurrent seizures and a clear cut diagnosis of epilepsy, especially if he or she has an identifiable syndrome with a predictable prognosis - for example, juvenile myoclonic epilepsy.

\section{CHOOSING TREATMENT}

Correspondence to: Professor DW Chadwick, Walton Centre for Neurology \& Neurosurgery, Lower Lane, Fazakerley, Liverpool L9 7LJ, UK d.w.chadwick@liv.ac.uk 
- Should seizures continue, the dose should be increased to the maximum tolerated before switching to alternative monotherapy. The seizure-free patient who is experiencing side effects will decide if the benefits of remission outweigh the symptoms.

- It is only when seizures continue despite adequate trials of two appropriate drugs that combination/dual therapy should be employed, there being little evidence that treatment with two drugs is superior to optimal treatment with one.

The aim of treatment is cessation of seizures without side effects. Pharmacological factors influencing the choice of drug include efficacy, toxicity, and ease of use, all of which should be considered with respect to individual patient needs.

\section{Efficacy}

There is considerable difficulty in comparing the relative efficacy of antiepileptic drugs (AEDs) because of the heterogeneity of epilepsy in samples in randomised clinical trials (RCTs). At one extreme are patients with mild epilepsy responding to most drugs in low dosage, and at the other extreme are those patients with a severe condition resistant to all medication, either singly or in combination.

\section{Partial onset seizures}

Although the evidence from individual RCTs is inconclusive, carbamazepine is probably the most effective drug for partial onset seizures. ${ }^{6}$ Certainly no drug has been shown to possess greater efficacy in these patients. Valproate and phenytoin are effective but concerns about their cosmetic effects and teratogenic potential limit their use in women of childbearing age.

Recent studies indicate the potential for newer drugs to become first line agents. Claims that lamotrigine and oxcarbazepine possess similar efficacy are poorly substantiated but they may be better tolerated. The place of gabapentin and topiramate as monotherapy remains uncertain.

\section{Generalised onset seizures}

Despite a lack of hard evidence from RCTs, sodium valproate is accepted as the drug with greatest efficacy, which presents a therapeutic dilemma in women of child bearing age. Ethosuximide is an alternative treatment for absences but is not effective against tonic-clonic seizures, a clinically important seizure type in adult generalised epilepsy syndromes. Experimental evidence predicts that lamotrigine and topiramate would possess a broad spectrum of efficacy; this is supported by placebo controlled trials of both drugs in Lennox-Gastaut syndrome and topiramate in newly diagnosed primary generalised tonic-clonic seizures.

\section{Toxicity}

With this information on relative efficacy of AEDs the choice of drug will largely be determined by side effect profiles. ${ }^{7}$ AEDs produce several different types of adverse effects.

Acute dose related toxicity is common and predictable, although the dose required to produce symptoms varies between individuals. Injudiciously rapid introduction of drugs is a common reason for apparent treatment failure. Symptoms resolve with reduction of dose. Several drugs (carbamazepine, phenytoin, phenobarbitone, benzodiazepines, lamotrigine) produce a non-specific encephalopathy manifested by diplopia, nystagmus, ataxia, dysarthria,
Table 1 Chronic anticonvulsant toxicity with antiepileptic drug treatment

\section{Nervous system \\ Memory and cognitive impairment, hyperactivity and behaviour disturbances, pseudodementia, cerebellar atrophy, peripheral neuropathy}

Ocular

Retinopathy

Skin

Acne, hirsutism, alopecia, chloasma

Liver

Enzyme induction

Blood

Megaloblastic anaemia, thrombocytopenia, pseudolymphoma

Immune system

IgA deficiency, drug induced systemic lupus erythematosus

Endocrine system

Decreased thyroxine concentrations, increased cortisol and sex hormone metabolism

Bone

Osteomalacia

Connective tissue

Gingival hypertrophy, coarsened facial features, Dupuytren's contracture

confusion, and drowsiness. Phenytoin rarely causes a dose dependent dyskinesia whereas an intermittent tremor is a common side effect of sodium valproate.

Acute idiosyncratic reactions are rare, unpredictable, and necessitate immediate withdrawal of the causative drug. Allergic reactions, manifested by rash with or without fever and other multi-organ involvement, occurs in $2-4 \%$ of patients exposed to carbamazepine, phenytoin, phenobarbitone or lamotrigine. Patients should be warned that even with cautious dosage escalation these reactions can occur within a few weeks of starting treatment with peak incidence at 10-21 days. Life threatening Stevens-Johnson syndrome or acute epidermal necrolysis occurs in 1:510000 but the risk may be higher in children. Aplastic anaemia is an extremely rare complication of phenytoin or carbamazepine treatment. Acute liver failure, attributable to valproate, is virtually confined to children under the age of 2 years who have additional neuropsychiatric problems, and may be caused by an inborn error of metabolism predisposing to this effect of the drug. The European Union licence for felabamate was withdrawn because of the significant risk of fatal haematological and hepatic reactions.

Chronic toxicity can affect any system (table 1). While some problems are caused by the known pharmacological properties of the drug-for example, macrocytic anaemia caused by folate deficiency (phenytoin)-others are, at present, unexplained-for example, weight gain (valproate) and weight loss (topiramate). Some effects, notably cosmetic changes, are cumulative and therefore long term, high dose polytherapy, especially with phenytoin and phenobarbitone, should be avoided.

These complications are usually discovered many years after licensing and their detection is an important aspect of long term surveillance. A retinopathy, ${ }^{8}$ manifested by a bilateral nasal or concentric visual field defect, which is usually asymptomatic, is now known to occur in $40 \%$ of patients exposed to vigabatrin treatment and guidelines restricting its use have been disseminated. 


\section{Teratogenicity}

Approximately $40 \%$ of patients with epilepsy are women of childbearing age, and women with epilepsy account for $0.5 \%$ of all pregnancies.

While the aetiology of congenital malformations is multifactorial, the risk is directly related to drug burden; monotherapy, dual therapy, and polytherapy carry risks of 4 $6 \%, 7-8 \%$, and $15-20 \%$, respectively. ${ }^{9}$

The risk of neural tube defects caused by carbamazepine is $0.5-1.0 \%$, while that associated with valproate is higher (at least $2 \%$ ) but probably dose related with a threshold of $1 \mathrm{~g}$ per day. A fetal anticonvulsant syndrome manifested by orofacial clefts, distal digital anomalies, and learning disability, with or without cardiac defects, has been attributed to several compounds.

Children with fetal valproate syndrome have characteristic dysmorphic features. Neither the incidence of this condition nor whether the physical features predict future learning difficulties are well understood.

While novel drugs are not recommended in pregnancy, thorough preclinical evaluation suggests that these compounds may be less teratogenic than established compounds. It is essential that the fetal risks of AEDs are better understood and all pregnancies in women receiving AEDs in the UK should be reported to the Epilepsy and Pregnancy Register in Belfast. ${ }^{10}$

It is generally agreed that, in most women, uncontrolled epilepsy presents a greater risk than drug treatment both to the mother and to the pregnancy. While guidelines on the management of pregnant women with epilepsy have been published in the specialist literature ${ }^{11}$ recent evidence reveals that this mostly takes place in the primary care setting where knowledge remains poor. Early counselling of women in childbearing years is essential and the epilepsy specialist nurse working within specific adolescent clinics is an ideal model for provision of information.

\section{Summary of choice of treatment}

At present carbamazepine and valproate are accepted as drugs of first choice for partial and generalised onset seizures, respectively. However, neither drug is free from side effects and there are particular concerns regarding the cosmetic and teratogenic effects of valproate. Several new drugslamotrigine, gabapentin, topiramate, oxcarbazepine-appear to be potential first line agents. Modern RCTs are designed to include composite outcome measures which include efficacy and tolerability, and to be large enough to produce statistically meaningful results. Whether the newer drugs possess any advantages is the focus of the ongoing SANAD (study of standard and new antiepileptic drugs) study.

\section{Monitoring treatment}

AED concentrations are over requested and often misinterpreted, leading to injudicious alteration of treatment.

Reasonable indications include:

- suspected poor or erratic compliance, especially in someone whose seizure control is much worse than expected;

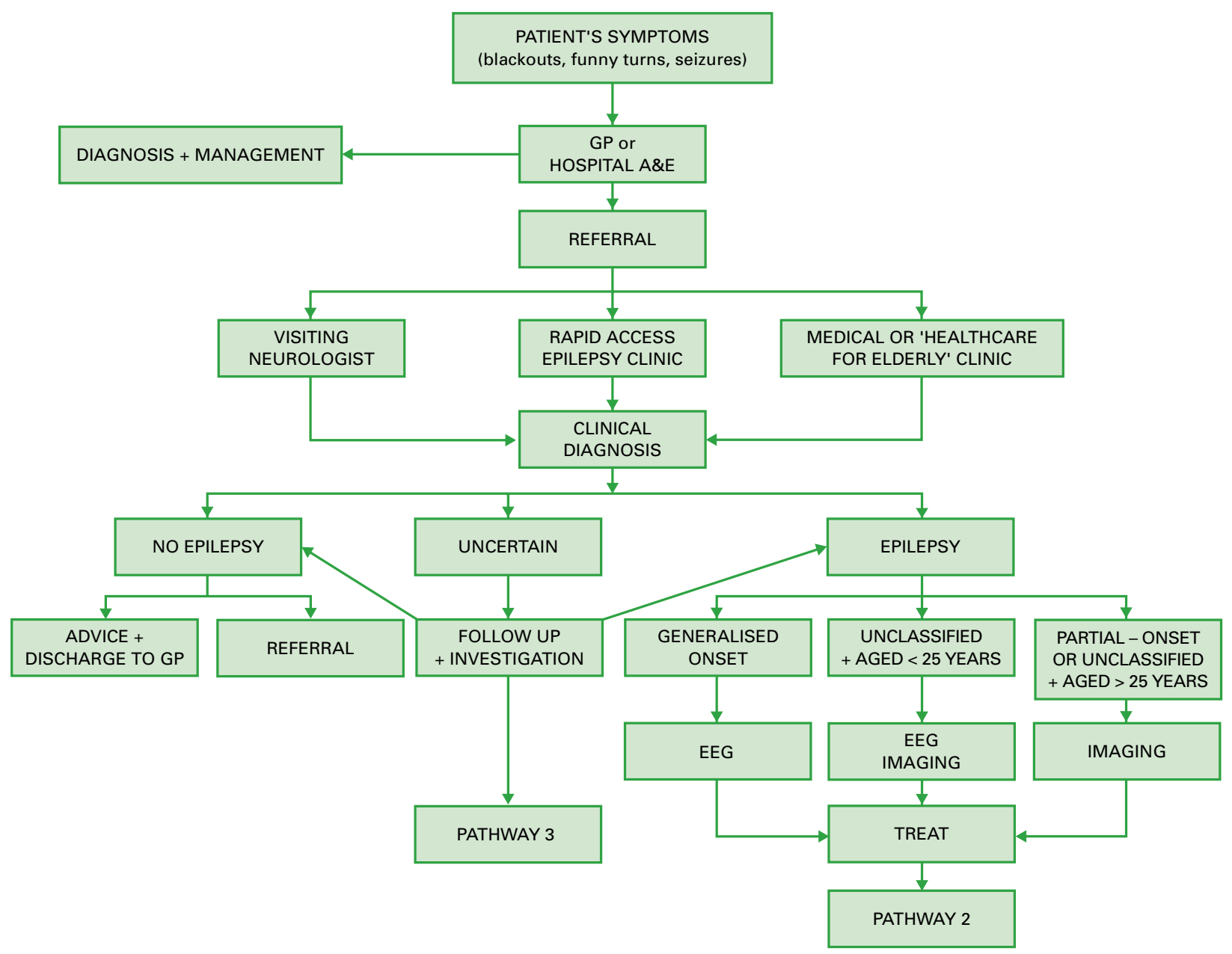

Figure 1 Pathway 1: diagnosis 
- symptoms of dose related toxicity where more than one drug could be the culprit-for example, intermittent diplopia, nausea, and ataxia in a patient receiving two membrane stabilising agents (phenytoin, carbamazepine, lamotrigine).

Similarly there is no place for routine haematology and biochemistry because the minor abnormalities encounteredfor example, raised transaminases caused by enzyme induction, thrombocytopenia on valproate, neutropenia and hyponatraemia on carbamazepine - are very rarely of clinical significance.

\section{A practical approach to management}

Figures 1 and 2 describe the diagnosis and early follow up in a multidisciplinary epilepsy clinic.

Visit 1 (consultant) -Following a confident clinical diagnosis of epilepsy, investigations to refine the diagnosis are requested and treatment is started. Classification, initially on clinical grounds, determines the choice of investigations. The idiopathic generalised epilepsies (IGEs) commence in childhood, adolescence, and early adult life. They are genetically determined conditions with generalised spike and wave frequently seen on the interictal EEG. The brain is structurally normal and imaging is not required. Epilepsy with onset in patients under 25 years of age, which cannot definitely be classified as partial or generalised, merits investigation with both EEG and imaging. Any epilepsy commencing over the age of 25 years of age demands the exclusion of structural pathology by imaging, but EEG may not be informative (over the age of 30-40 years). The patient should receive information about the diagnosis, the purpose of investigation, the most common side effects of treatment, any driving regulations, and occupational implications. There should be early contact with a specialist nurse who becomes the primary provider of information and care.

Visit 2 (3-4 months, nurse)-The patient receives results of investigations and more comprehensive counselling tailored to his or her own needs. This particularly applies to women of childbearing age. Patients who are seizure-free with no side effects can be given an optimistic prognosis. In cases

\section{Summary 1}

- The optimal management of patients with epilepsy requires cooperation between specialist, GP, and patient. Patients achieving remission can be discharged to GP care while others require specialist follow up

- Most patients with a first seizure do not need treatment but there are individual exceptions

- When initiating treatment a "start low, go slow" approach reduces risk of intolerance

- Carbamazepine and valproate are standard drugs of first choice for partial and generalised onset seizures, respectively. Whether novel AEDs confer benefits that justify their increased cost is the focus of ongoing research

- The management of women with idiopathic generalised epilepsies who want children presents a real dilemma

- All women with epilepsy who become pregnant should be asked to contact the UK Epilepsy and Pregnancy Register in Belfast

where seizures continue or side effects are troublesome, a management plan is formulated in consultation with the clinician and subsequently supervised by the nurse.

Visit 3 (12 months or earlier in patients with active epilepsy, consultant)-Around $60-70 \%$ of patients will be in remission. The need for long term treatment is discussed. Patients are informed that if they achieve a two year remission they can be offered an individualised estimate of the risk of relapse on drug withdrawal. ${ }^{12}$ They can be discharged to their GP with advice on reasons for re-referral. The remaining 30-40\% require further follow up in specialist clinic.

\section{Stopping treatment}

The decision to stop medication involves a balance of the risks of continuation (chronic toxicity, teratogenicity) with the implications of relapse (injury, SUDEP, loss of driving licence, effect on job).

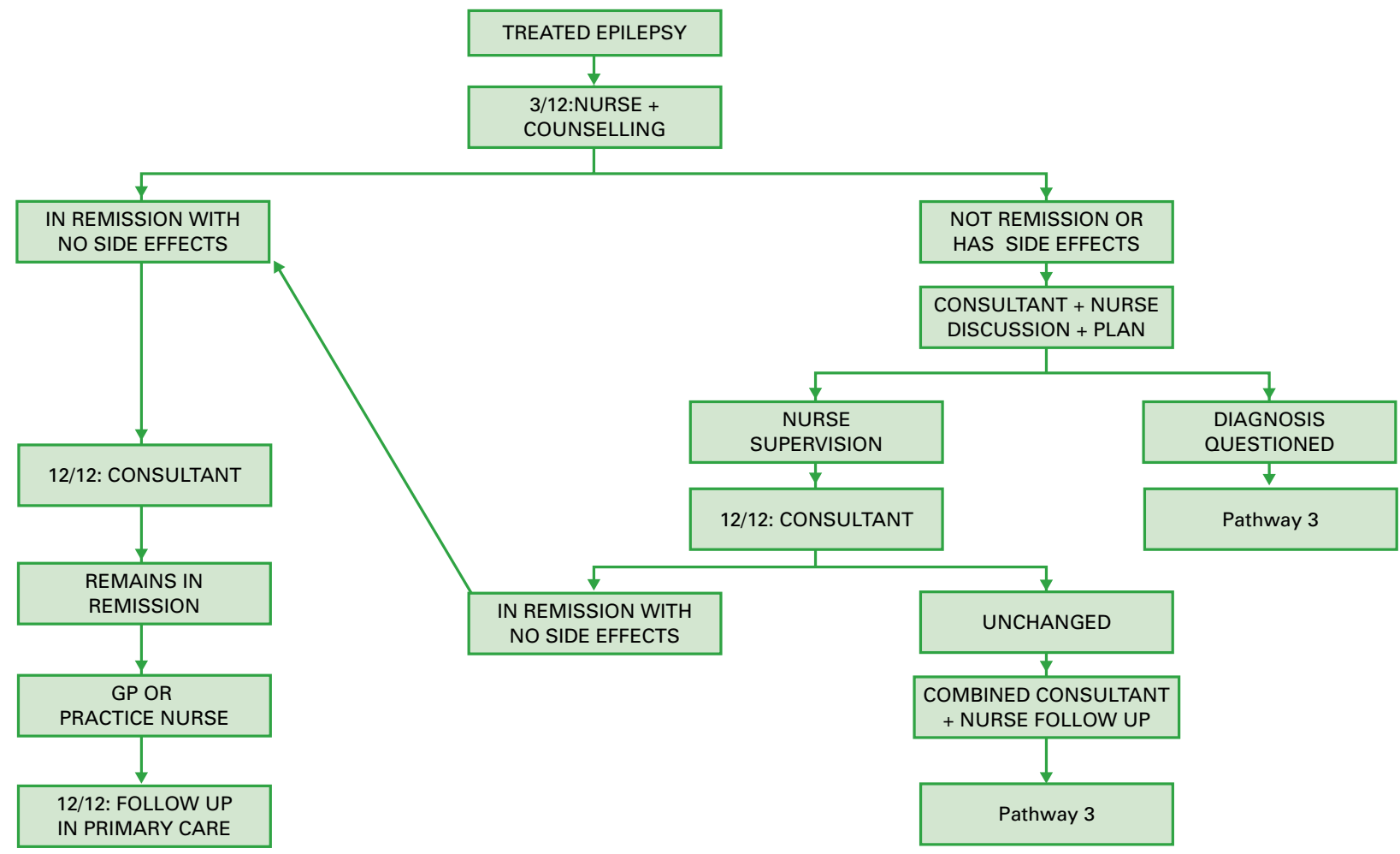

Figure 2 Pathway 2: management and follow up (treated epilepsy) 
It is accepted that withdrawal should be considered after a two year remission. While paediatricians usually recommend withdrawal, adult neurologists adopt a more circumspect approach.

In a heterogenous group of patients with epilepsy in remission, planned withdrawal doubles the risk of relapse at two years $(40 \% v 20 \%)$. The other important predictors of risk are number of drugs (one or more than one), whether or not seizures have occurred since starting treatment, duration of remission, seizure type (tonic-clonic, myoclonic, other), and EEG (normal, epileptiform abnormality). ${ }^{12}$ Using this information individualised estimates of risk of relapse can be calculated permitting informed decision making. Most patients who drive elect to stay on treatment.

\section{Refractory epilepsy}

Figure 3 describes a strategic approach to management of patients who have not responded to treatment or who have been referred with a drug resistant epilepsy.

\section{Review the diagnosis}

The accuracy of diagnosis and classification should be carefully reviewed. Around $15 \%$ of patients will not have epilepsy while $10-20 \%$ of those with epilepsy may not have received first choice treatment. ${ }^{13}$

Patients with non-epileptic attacks (pseudoseizures) ${ }^{14}$ can usually be identified on clinical grounds. Women predominate and psychiatric comorbidity is almost universal. Chronic depression, dissociative states consequent upon physical, sexual or emotional abuse, and previously unexplained physical symptoms (somatisation) are common findings. Frequent hospitalisation, in apparent status epilepticus, is also common. Video-telemetry helps to confirm the diagnosis and can also identify seizures of frontal origin and patients with both epilepsy and pseudoseizures.
When the diagnosis of epilepsy is removed, AEDs are stopped and further intervention is tailored to the patients' needs. Management of this heterogenous group of patients requires involvement of both the neuropsychiatrist, for diagnosis of specific, treatable underlying disorders, and the neuropsychologist, for identification and treatment of factors predisposing to this behaviour.

\section{Review the classification of epilepsy}

Each patient's epilepsy should be syndromically classified ${ }^{15}$ using existing evidence. Previously unrecognised IGE, notably juvenile myoclonic epilepsy, not treated with valproate, is the most common example of suboptimal treatment.

Where classification is uncertain further investigation is needed. Clinically the distinction between IGE, unresponsive to valproate, and frontal lobe epilepsies, can pose difficulties. Tonic-clonic and complex partial seizures of frontal lobe origin often occur without warning. Seizures, indistinguishable from typical absences, can arise from the mesial frontal lobe and electrical stimulation of this area can produce generalised spike and wave on scalp electrodes. Similarly brief, asymmetric tonic seizures and bilateral clonic movements of upper limbs, without loss of consciousness, arising from the supplementary motor area, can be mistaken for generalised myoclonus. Video-telemetry may be required to classify the disorder. In patients with partial seizures high definition magnetic resonance imaging (MRI) may reveal structural pathology amenable to surgery.

\section{Identify/exclude structural pathology}

Refractory partial epilepsy demands exclusion of structural pathology. It is worthwhile confirming that previous imaging reports are accurate. MRI has a higher diagnostic yield in these patients. Some findings (for example, diffuse low grade glioma, multifocal malformation of cortical development) may have no

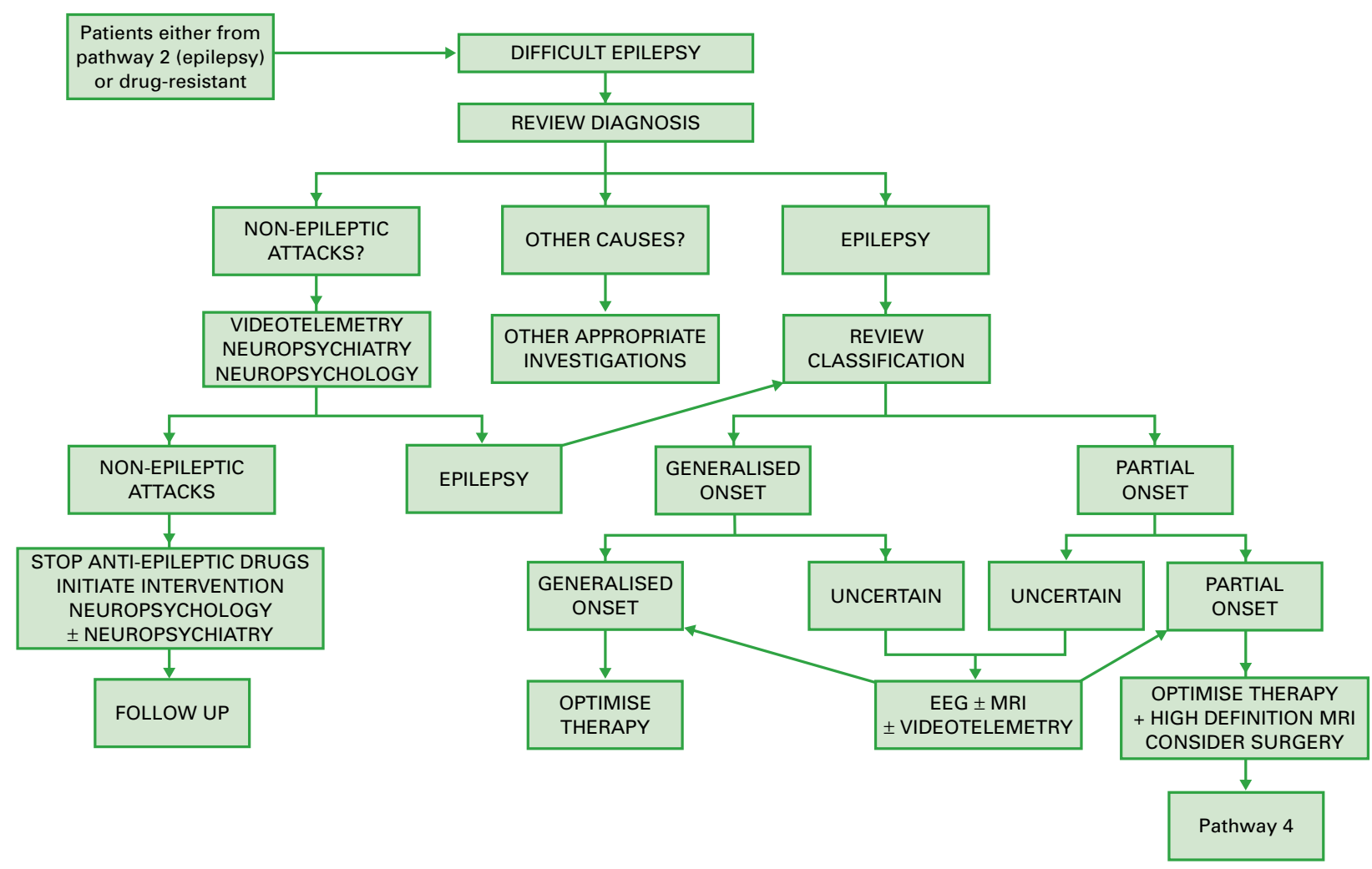

Figure 3 Pathway 3: difficult epilepsy. 
ii20

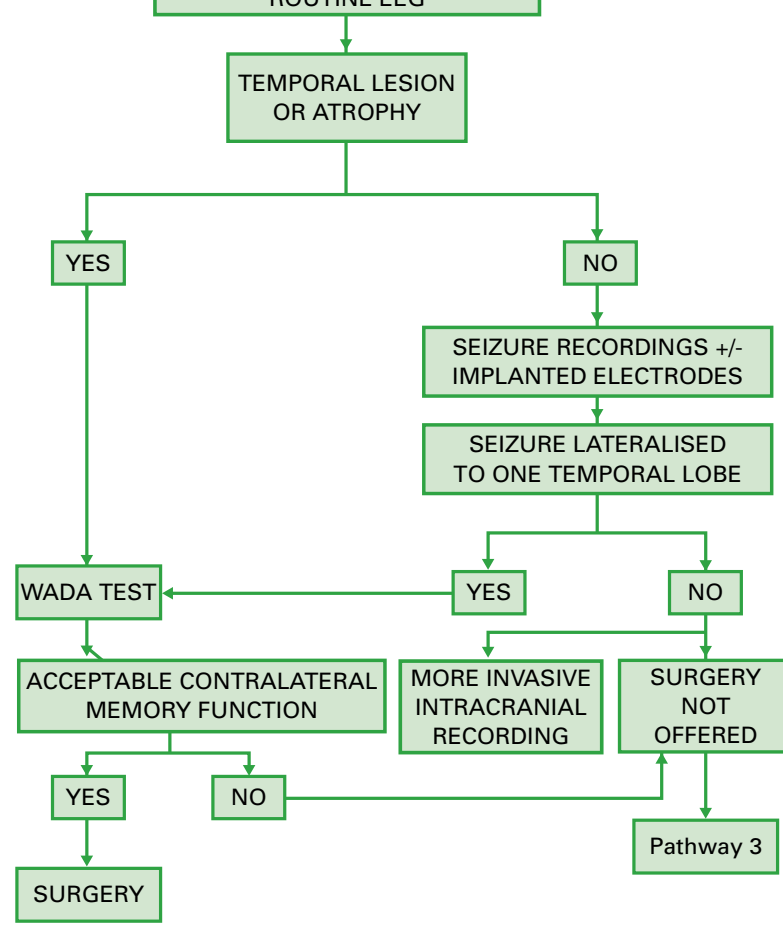

Figure 4 Pathway 4: pre-surgical evaluations.

therapeutic implications but explain the refractory nature of that patient's epilepsy. Occasionally unexpected vascular disease prompts a search for a treatable underlying cause-for example, antiphospholipid syndrome.

\section{Consider surgery}

Some patients will be candidates for surgery. ${ }^{16}$ The standard MRI may have identified resectable pathology. In addition all those thought to have seizures of temporal lobe origin, who are prepared to accept risks of investigation and surgery, should be evaluated at the earliest opportunity.

Figure 4 is an example of a modern pre-surgical investigation protocol which places emphasis on a non-invasive approach. The presence (or absence) of unilateral atrophy on high definition MRI is an excellent predictor of both suitability for surgery and the outcome thereof; patients can therefore be counselled accordingly at an early stage. An ideal candidate has a history of prolonged early febrile convulsion(s), medial temporal seizures (rising epigastric sensation followed by trance with lip smacking automatisms), and concordance between interictal EEG (focal sharp/spike), high definition MRI (atrophy), and Wada test (impaired ipsilateral memory). The processing of such cases should be possible in every neuroscience centre in the UK. Furthermore, limiting surgery to these straightforward cases should ensure seizure-free rates approaching $80 \%$ balanced against complication rates of less than $1 \%$ mortality and 3-4\% permanent neurological deficit.

More difficult cases will require semi-invasive (foramen ovale) or invasive (subdural, depth) intracranial monitoring in specialist centres. Even if seizures are thought to be localised the prospects for surgical success are lower and the total risks of investigation and surgery are higher, and this should be reflected in patient counselling.

Summary 2
- AED withdrawal can be considered after a two year
remission but patients should be allowed to make
informed decisions
- Adopt a strategic approach to patients with refractory
epilepsy
- Patients with non-epileptic attacks should be handled
sensitively; a multidisciplinary approach is essential
- Give early consideration to surgery in patients with drug
resistant temporal lobe epilepsy. Unilateral atrophy on
high definition MRI is a good predictor of suitability for
surgery and outcome
- Avoid polytherapy, or combinations of drugs with similar
side effect profiles
- Set realistic goals and resist temptation to make repeated
treatment changes in patients with chronic but stable
conditions

Some patients who are unsuitable for resective surgery may benefit from vagus nerve stimulation. ${ }^{17}$ Clinical trials indicate that this procedure is well tolerated, has no serious side effects, and reduces seizure frequency by $50 \%$ or more in a third of patients. More dramatic improvement has been observed in individual cases. Preliminary findings suggest that benefit is sustained.

\section{Drug treatment of refractory epilepsy}

For the majority of patients medical treatment remains the only option. The aims are minimisation of the number of tonic-clonic seizures and side effects by avoiding polypharmacy, and limiting disability associated with the psychosocial consequences of the chronic disorder.

Most of the newer drugs are easy to use. ${ }^{18}$ As a group they produce sustained benefit, in terms of reduced seizure frequency or severity, in a minority of patients. There is some evidence that topiramate may be the most potent. Lamotrigine and gabapentin are generally well tolerated but combination with high doses of carbamazepine can cause cerebellar side effects. The GABA-ergic compounds (topiramate, tiagabine) not uncommonly cause cognitive and/ or mood disturbance, and should be used with caution in patients with significant psychiatric problems. It is hoped that thorough preclinical evaluation predicting low risks of teratogenicity will be borne out in clinical practice in humans.

If monotherapy fails and a combination of drugs is used then agents effective for the type of epilepsy with different modes of action and low risk of pharmacokinetic interaction are preferred because of the theoretical possibility of synergy and, more importantly, a lower chance that side effects will be additive.

For generalised onset seizures a combination of valproate and lamotrigine, or failing this, topiramate should be used. Ethosuximide remains an option for absences.

For partial onset seizures two drugs-carbamazepine or another sodium channel blocker (phenytoin or lamotrigine) and another drug with a different mode of action-should be used. Thus combinations of carbamazepine with phenytoin and lamotrigine are best avoided. While polytherapy should be avoided, the intermittent use of clobazam for predictable clustering, notably perimenstrually, is sometimes worthwhile. When epilepsy is refractory but stable it is usually better to accept the situation than to indulge in repeated treatment changes with unpredictable outcomes. These patients can often be discharged to the care of their GP. 


\section{References}

1 Dulac O, Leppik I. Initiating and discontinuing treatment. In: Engel J Jr, Pedley TA, eds. Epilepsy: a comprehensive textbook. Philadelphia: Lippincott-Raven, 1997:1237-46.

2 Leestma JE, Annegers JF, Brodie MA, et al. Sudden unexplained death in epilepsy: observations from a large clinical development programme. Epilepsia 1997;38:47-55.

- Reviews the subject and identifies overall risk and factors associated with greater risk of SUDEP.

3 Schierhout G, Roberts I. Prophylactic antiepileptic agents after head injury: a systematic review. J Neurol Neurosurg Psychiatry 1998;64:108-12.

- Clearly demonstrates that long term prophylactic antiepileptic drug treatment after head injury is not indicated.

4 Berg AT, Shinnar S. The risk of recurrence following a first unprovoked seizure. Neurology 1991;41:965-72.

5 Musicco M, Beghi E, Solari A, et al. Treatment of first tonic-clonic seizure does not improve the prognosis of epilepsy. First seizure trial group (FIRST group). Neurology 1997;49:991-8.

6 Marson AG, Williamson PR, Hutton JL, et al on behalf of the Epilepsy Monotherapy Trialists. Carbamazepine versus valproate monotherapy for epilepsy. In: The Cochrane Library Issue 4, 2000. Oxford: Update Software.

- Rigorous meta-analysis of all relevant trials.

7 Timmings P. Toxicity of antiepileptic drugs. In: Engel J Jr, Pedley TA eds. Epilepsy: a comprehensive textbook. Philadelphia: Lippincott-Raven, 1997:1165-74.
8 Eke T, Talbot JF, Lawden MC. Severe persistent visual field constriction associated with vigabatrin. BMJ 1997;314:180-1.

9 Zahn C. Neurological care of women with epilepsy. Epilepsia 1998;39(suppl 8):S26-31.

10 Morrow JI, Craig JJ, Russell AJC, et al. The UK Epilepsy and Pregnancy Register. Epilepsia 2000;41(suppl):142.

- This long overdue work will inform future practice for the benefit of women with epilepsy. All relevant patients should be registered.

11 Crawford P, Appleton RA, Betts T, et al. Best practice guidelines for management of women with epilepsy. Seizure 1999;8;201-17.

12 Chadwick D, and the MRC AED Withdrawal Study Group. Randomised study of AED withdrawal in patients in remission. Lancet 1991;337:1175-80.

- A pivotal study. The data permit individualised counselling of patients assisting informed decision making.

13 Smith D, Defalla BA, Chadwick DW. The misdiagnosis of epilepsy and the management of refractory epilepsy in a specialist clinic. QJM 1999;92:15-23.

14 French JA. Pseudoseizures in the era of video-electroencephalogram monitoring. Curr Opin Neurol 1995;8:117-20.

15 Commission on Classification and Terminology of the International League Against Epilepsy. Proposal for revised classification of epilepsies and epileptic syndromes. Epilepsia 1989;30:389-99.

16 Engel J Jr. Surgery for seizures. N Engl J Med 1996;334:647-52.

- Excellent review of a complex subject.

17 Schachter SC, Saper CB. Vagus nerve stimulation. Epilepsia 1998;39:677-86.

18 Marson AG, Kadir ZA, Hutton JL, et al. The new antiepileptic drugs: a systematic review of their efficacy and tolerability. Epilepsia 1997;38:859-80.

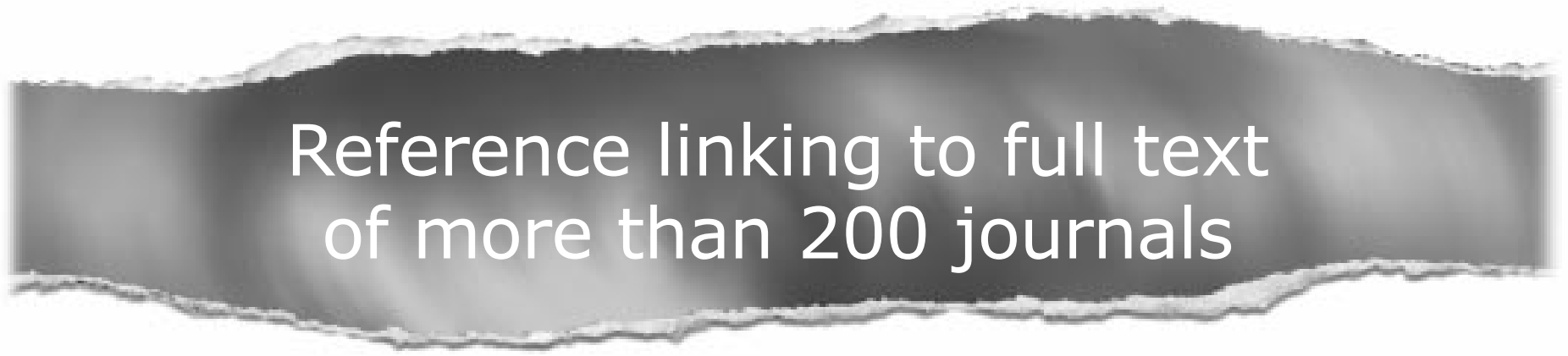

\section{Toll free links}

You can access the FULL TEXT of articles cited in the Journal of Neurology, Neurosurgery, and Psychiatry online if the citation is to one of the more than 200 journals hosted by HighWire (http://highwire.stanford.edu) without a subscription to that journal. There are also direct links from references to the Medline abstract for other titles.

\section{www.jnnp.com}

